\title{
Uterine scoring system for reproduction scoring correlation with pregnancy rate in infertility patients undergoing intracytoplasmic sperm injection and embryo transfer
}

\author{
Mamta Vijay Shivtare ${ }^{1 *}$, Nikita Lad ${ }^{1}$, Pallavi Vishwekar ${ }^{2}$, Pradnya Shetty ${ }^{1}$
}

\author{
${ }^{1}$ Department of IVF, D. Y. Patil Medical College, Navi Mumbai, Maharashtra, India \\ ${ }^{2}$ Department of Obstetrics and Gynecology, D. Y. Patil Medical College, Navi Mumbai, Maharashtra, India
}

Received: 18 February 2019

Accepted: 08 April 2019

\section{*Correspondence:}

Dr. Mamta Vijay Shivtare,

E-mail: drmamta84@gmail.com

Copyright: () the author(s), publisher and licensee Medip Academy. This is an open-access article distributed under the terms of the Creative Commons Attribution Non-Commercial License, which permits unrestricted non-commercial use, distribution, and reproduction in any medium, provided the original work is properly cited.

\begin{abstract}
Background: Infertility is defined as failure to conceive a clinical pregnancy after 12 months or more of regular unprotected intercourse. The birth of Louis Brown opened door to whole new world. Science of reproduction unfolded with better understanding of physiology and pharmacology of gonadotropins. Improved stimulation protocol, and lab facilities are available for IVF and intracytoplasmic sperm injection (ICSI). The objective of this study was to study relation between uterine scoring system for reproduction (USSR) scoring and pregnancy rate in patients undergoing ICSI and embryo transferred.

Methods: A prospective observational study was conducted. Study comprised 48 women visiting with infertility for ICSI and embryo transfer. Baseline scan done on day2 of menses. Patients started on estradiol valerate for endometrial preparation. Transvaginal sonography done on $10^{\text {th }}$ day of menstrual cycle for USSR scoring. USSR scoring includes parameters such as endometrial thickness, endometrial layering, myometrial contractions, myometrialechogenecity, uterine artery Doppler flow, endometrial blood flow, myometrial blood flow.

Results: Out of total 48 infertility patients for embryo transfer 22 conceived, which gives $45.83 \%$ pregnancy rate. No patients had a perfect score of 20. Patients with score of 17-19 had pregnancy rate of $66 \%$. Endometrial thickness of $10-14 \mathrm{~mm}$ gave optimum result of $52 \%$. Pulsatality index $<2.19$ was associated with pregnancy rate of $66 \%$.

Conclusions: USSR scoring is highly indicative of good pregnancy outcome in patients undergoing ICSI and embryo transfer. Endometrial morphology and thickness was strongly correlated with successful pregnancy outcome.
\end{abstract}

Keywords: USSR scoring, Embryo transfer, ICSI, TVS

\section{INTRODUCTION}

Infertility is defined as failure to conceive a clinical pregnancy after 12 months or more of regular unprotected intercourse. The birth of Louis Brown opened door to whole new world. Science of reproduction unfolded with better understanding of physiology and pharmacology of gonadotropins. Improved stimulation protocol, and lab facilities are available for IVF and ICSI. Better ultrasound and Doppler studies of uterus and endometrium have been used. Successful implantation requires presence of a healthy embryo and receptive endometrium. Endometrial receptivity is ability of endometrium to successfully attach embryo, nourish and keep it alive.

Endometrium undergoes clinical, morphological and histological changes with typical sonography patterns 
during different phases of natural as well as stimulated cycles. From the first day of menstrual cycle till mid cycle endometrium progressively thickens and it is sonographically detectable. This appearance can be layered, trilamilar or five line. A good blood supply to the endometrium is considered essential for implantation and hence assessment of endometrial blood flow in embryo transfer cycle patients has gained importance over years. Endometrial and subendometrial blood flow increases during follicular phase and reach its maximum approximately three days prior to ovulation. Endometrial blood flow can be measured by $2 \mathrm{D}$ or $3 \mathrm{D}$ ultrasound.

Spiral vessels run towards endometrium and penetrate it. These spiral arteries are branches of uterine artery and therefore if resistance in uterine artery is high, evidently resistance in spiral arteries is also high. So assessment of uterine artery resistance is a clue to implantation potential of endometrium. Estrogen produces vasodilatory effect on uterine artery. So pulsatality index drops with increasing estrogen levels. Transvaginal sonography is a simple, reliable and inexpensive method for evaluation of endometrial receptivity. These sonographic findings are weighed according to uterine scoring system for reproduction (USSR) or uterine biophysical profile (UBP) by $M$. Applebaum. It has become absolute necessity to evaluate uterus and endometrium prior to embryo transfer, so that optimum results are obtained in favorable uteri.

Objective of this study was to evaluation of USSR scoring in infertile women for ICSI and embryo transfer cycles. To study relation between USSR scoring and pregnancy rate in ICSI and embryo transfer patients.

\section{METHODS}

Study comprised of women visiting with infertility to IVF department. Hospital based prospective observational study was conducted. A total of 48 women with infertility undergoing ICSI and embryo transfer were analyzed. Study was conducted between July 2018 to September 2018. Age of patients was between 25 to 40. Duration of infertility was 2 to 10 years. Initial work up was done with investigations. Patients undergoing frozen embryo transfer, oocyte donation cylcles and embryo donation cylces were included. Male factor infertility patients were excluded.

Patients for frozen embryo transfer were pretreated with suppression by OC pills and GnRH agonist followed by antagonist protocol for controlled ovarian stimulation. These patients were started on OC pills in previous month and Inj. Lupride depot $3.75 \mathrm{mg}$ given 7 days prior to stoppage of OC pills. Baseline scan was done on day 2 of menses. Injection Human Menopausal Gonadotropin started and continued until leading follicle size of $14 \mathrm{~mm}$. Antagonist Injection Ciscure $0.25 \mathrm{mg}$ started at this stage. Trigger given when leading follicle size more than 18 $\mathrm{mm}$ and 3 or more follicles of size 16 to $18 \mathrm{~mm}$. Oocyte retrieval was done 36 hours after trigger under SGA.

Patients with frozen embryo transfer, oocyte donation cycles and embryo donation cycles were treated with Estradiol Valerate. Baseline transvaginal sonography on day 2 of menses in lithotomy position after emptying bladder completely for endometrial thickness and to rule out any ovarian pathology and suppression. Estradiol started at the dose of $2 \mathrm{mg}$ twice a day and adjusted according to sonography findings. TVS was repeated on day 10 and then alternate days. USSR scoring done four days prior to embryo transfer. Progesterone started on same day for three days till embryo transfer. Embryo transfer done with 2 to 3 good quality embryos. Luteal phase support given. Sr B- hCG done on 14th day following embryo transfer.

\section{Endometrium}

Smith's Grading 8

Grade A: bright endometrium - post ovulation or the luteal phase.

Grade B: endometrial reflectivity is similar to myometrium - late follicular phase.

Grade C: a solid area of reduced reflectivity appears as a dark area next to the lighter myometrium - mid follicular phase.

Grade D: echoes are absent in endometrium but a bright central echo is seen, described as he triple line.

In late follicular phase, grade B pattern is associated with higher pregnancy rate

\section{Endometrial thickness and pattern in menstrual cycle}

- Menstrual phase - $<5 \mathrm{~mm}$ - thin

- Early follicular phase (day 6-10) - 7 to $9 \mathrm{~mm}$ - distinct triple line hyperechogenic endometrium.

- Late follicular phase ( day 11 to ovulation) - 9 to 12 $\mathrm{mm}$ - isoechogenic (similer to myometrium)

- Luteal phase (post ovulation) - 10 to14 mm - bright fluffy, absence of triple line.

\section{Endometrial zones}

Depending upon vascularization of endometrial layers, late proliferative endometrium is divided into four number zones. ${ }^{6,8}$

- Zone I: $2 \mathrm{~mm}$ thick area surrounding zone II.

- Zone II: Hyperechoeic outer endometrial edge.

- Zone III: Hyperechoeic inner endometrial edge.

- Zone IV: Endometrial cavity. 
Applebaum uterine scoring system for reproduction (USSR) or uterine biophysical profile (UBP). ${ }^{1,6,8}$

It is based on sonographic features of uterus during midcycle, includes following parameters:

- Endometrial thickness: Maximum distance between echogenic interface of myometrium and endometrium, measured along longitudinal axis of uterus.

- Endometrial layering: Five distinct layers.

- Endometrial motion: Myometrial contractions within 2 minutes causing wave like motions of endometrium.

- Myometrial echogenicity

- Uterine artery Doppler flow: Pulsatility index. (PI): impedence of blood flow through uterine arteries PI can be calculated as peak systolic velocity (PSV) minus end diastolic velocity divided by the mean.

- Endometrial blood flow within zone III

- Myometrial blood flow seen on gray scale examination internal to the arcuate vessels

Table 1: Scoring of each parameter. ${ }^{8}$

\begin{tabular}{|c|c|}
\hline Parameter & Scoring \\
\hline \multirow{4}{*}{$\begin{array}{l}\text { Endometrial } \\
\text { thickness: }\end{array}$} & $<7 \mathrm{~mm}=0$ \\
\hline & $7-9 \mathrm{~mm}=2$ \\
\hline & $10-14 \mathrm{~mm}=3$ \\
\hline & $>14 \mathrm{~mm}=1$ \\
\hline \multirow{3}{*}{$\begin{array}{l}\text { Endometrial } \\
\text { layering: }\end{array}$} & No layering $=0$ \\
\hline & Hazy 5 line = 1 \\
\hline & Distinct 5 line $=3$ \\
\hline \multirow{2}{*}{$\begin{array}{l}\text { Endometrial } \\
\text { motion: }\end{array}$} & $<3$ contractions in 2 minutes $=0$ \\
\hline & $>3$ conractions in 2 minutes $=3$ \\
\hline \multirow{2}{*}{$\begin{array}{l}\text { Myometrial } \\
\text { echogenicity: }\end{array}$} & Coarse, inhomogeneous $=1$ \\
\hline & Relatively homogenous $=2$ \\
\hline \multirow[t]{4}{*}{5.} & $>3=0$ \\
\hline & $2.5-2.99=0$ \\
\hline & $2.2-2.49=1$ \\
\hline & $<2.19=2$ \\
\hline \multirow[t]{3}{*}{6.} & Absent $=0$ \\
\hline & Sparse blood flow $=2$ \\
\hline & Multifocal blood flow $=5$ \\
\hline \multirow{2}{*}{$\begin{array}{l}\text { 7. Myometrial } \\
\text { blood flow } \\
\text { on gray scale } \\
\text { examination: }\end{array}$} & Absent $=0$ \\
\hline & Present $=2$ \\
\hline
\end{tabular}

\section{RESULTS}

Total numbers of patients included in study were 48 with age between 25 to 40 and duration of infertility from 2 to 10 years. Out of 48 women included in the study 22 conceived which a pregnancy rate of $45.83 \%$. No patients had a perfect score of 20.15 patients had a score of 17 19 of which 10 conceived giving pregnancy rate of $66 \%$.
22 patients with score of 14-16 of which 10 conceived with pregnancy rate of $45 \%$ and 11 patients with score $<13$ of whom only $18 \%$ conceived..$^{3,6,7}$ Pregnancy relation with USSR score is depicted in Table 2.

Table 2: USSR Scoring Relation with pregnancy.

\begin{tabular}{|llll|}
\hline Score & No. & Pregnant & $\%$ of pregnancy \\
\hline 20 & 0 & 0 & $0 \%$ \\
\hline $17-19$ & 15 & 10 & $66 \%$ \\
\hline $14-16$ & 22 & 10 & $45 \%$ \\
\hline$<13$ & 11 & 2 & $18 \%$ \\
\hline Total & $\mathbf{4 8}$ & $\mathbf{2 2}$ & $\mathbf{4 5 . 8 3 \%}$ \\
\hline
\end{tabular}

Endometrial thickness in maximum patients was in the range of 7-9mm with 23 patients showing pregnancy rate of $47 \%$. Highest pregnancy rate of $52 \%$ was seen with thickness of $10-14 \mathrm{~mm}$. Endometrial thickness more than $14 \mathrm{~mm}$ reduced pregnancy rate to $0 \%$. Multifocal endometrial blood flow in zone III is associated with $66 \%$ conception. Sparse blood flow was present in 34 patients of which 14 were pregnant. ${ }^{3,9}$ Absent endometrial blood flow is associated with no conception. Multifocal blood flow gave highest conception rate i.e. $66 \%$.Homogenous Myometrium with good blood flow shows success results in $75 \%$.

Maximum patients had PI between 2.2-2.49 with conception rate of $51.42 \%$. PI values of $2.5-2.99$ had a success rate of only $25 \%$, illustrated in Figure 1.,2,11 Pulsatality index of more than 3 was present in 3 patients with no pregnancy. PI less than 2.15 is favourable for pregnancy giving success rate of $66 \%$. So a total pregnancy rate of $45.83 \%$. maximum pregnancy seen with score of $17-19$, for thickness of $10-14 \mathrm{~mm}$ i.e. $52 \%$, with multifocal blood flow in zone III and IV, with distinct five line appearance of endometrium which is $66 \%$ and with PI $<2.19$ with pregnancy percentage of $66 \%$.

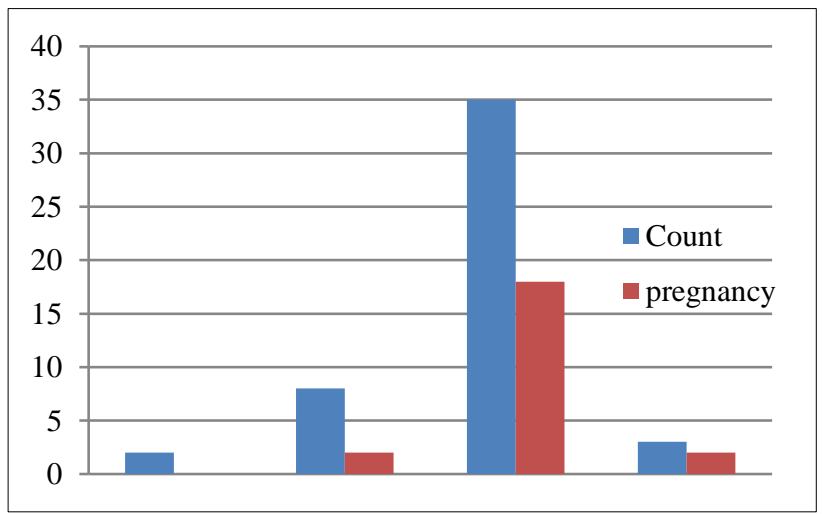

Figure 1: Pulsatality index (PI).

\section{DISCUSSION}

USSR scoringis highly indicative of good pregnancy outcome in patients undergoing embryo transfer. We 
found optimum pregnancy results with a score of 17-19. Assessment of endometrial receptivity is mandatory for success of ICSI and IVF

In this study endometrial thickness and morphology was strongly correlated with successful pregnancy. ${ }^{9-11}$ Most suitable endometrial thickness was $10-14 \mathrm{~mm}$, followed by $7-9 \mathrm{~mm} .{ }^{5}$ Endometrium $<6 \mathrm{~mm}$ is associated with no conception. We found decreased pregnancy rate in patients with endometrial thickness more than $14 \mathrm{~mm}$. Implantation and pregnancy rate may be adversely affected by thick endometrium. Distinct five line or triple layer pattern of endometrium shows better implantation rate as compared to hazy, homogenous endometrium shown in Table $3 .{ }^{4,5}$

Table 3: Endometrial layering.

\begin{tabular}{|llll|}
\hline Findings & No. & Pregnancy & $\%$ of pregnancy \\
\hline No layering & 02 & o & 0 \\
\hline Hazy 5 line & 30 & 12 & $40 \%$ \\
\hline Distinct 5 line & 16 & 10 & $62 \%$ \\
\hline
\end{tabular}

Endometrial thickness and subendometrial blood flow patterns are useful prognostic parameters for successful pregnancy. Endometrial blood flow was directly correlated with pregnancy outcome following embryo transfer. Multifocal blood flow in zone III and IV had maximum pregnancy rate. Similar findings are seen in study by Singh et al. ${ }^{12}$

Patients with low uterine artery PI on day of embryo transfer were more likely to conceive than those with high PI. Hence lower PI is associated with better outcome of pregnancy. ${ }^{2,3,11}$ Raised PI is associated with impaired uterine blood flow, which hampers implantation while optimum uterine blood perfusion gives good results.

\section{CONCLUSION}

Applibaum's USSR scoring is a simple and noninvasive method for prediction of pregnancy rate in ICSI and embryo transfer cycle. Endometrial receptivity including thickness and vascularity are predominant in predicting outcome of pregnancy.

Funding: No funding sources Conflict of interest: None declared

Ethical approval: The study was approved by the Institutional Ethics Committee

\section{REFERENCES}

1. Applebaum M. The uterine biophysical profile in Allahabadia G(Ed). Endosonography in Obstetrics and Gynaecology, Mumbai. Rotunda Medical technologies 1td. 1997:343-52.

2. Zaki H, Geneidi E, Coulam C. Revisiting predictive value of uterine aartery pulsatality index for uterine receptivity. Fertnstert. 2015;07:1065.

3. Malhotra N, Malhotra J, Singh A, Gupta P, Malhotra $\mathrm{N}$. Endometrial receptivity and scoring for prediction of implantation and newer markers. J South Asian Feder Obst Gynae. 2017;9(2):143-54.

4. Heger. Endometrial receptivity and its predictive value for IVF or ICSI outcome. Geburtshilfe Frauenheilkd. 2012;72(8):710-5.

5. Khan MS, Khan A, Ratnani R. USG and Doppler study to predict uterine receptivity in infertile patients undergoing embryo transfer. J Obstel Gynaecol India. 2016;66 (suppl1):377-82.

6. M. Applebaum The Uterine Biophysical Profile. Ultrasound Obstet Gynecol. 1995.

7. Mukhopadhyay I. Prediction of outcome of conception cycle by TVS with help of USSR and UBP method in West Bengal. Int J Rec Sci Res. 2015;6(5):4114-6.

8. Rao KA, Deepika monitoring of ovarian stimulation. TVS \& Doppler in infertility patients 472-477 Principles and fassisted reproductive technology. $3^{\text {rd }}$ Ed.; 646-649.

9. Sardana D, Upadhyay A, Deepika K, Gautam T, Pranesh, Rao KA. Correlation of subendometrial and endometrial blood flow with 2D Doppler with pregnancy outcome in FET cycles. J Hum Reprod Sci. 2014;7(2):130-5.

10. Chen SL, Wu FR, Luo C, Chen X, Shi XY, Zheng $\mathrm{HY}$, Ni YP. Combined analysis of endometrial thickness and pattern in predicting outcome of in vitro fertilization and embryo transfer: a retrospective cohort study. Repro Biol Endocrinol. 2010;8(1):30.

11. Dechaud H, Bessueille E, Bousquet PJ, Reyftmann L, Hamamah S, Hedone B. Optimal timing of ultrasonographic and Doppler evaluation of uterine receptivity to implantation. Reproductive Biomed Online. 2008;16(3):368-75.

12. Singh N, Bahadur A, Mittal S, Malhotra N, Bhatt A. Predictive value of endometrial thickness, pattern and sub-endometrial blood flows on the day of hCG by $2 \mathrm{D}$ doppler in in-vitro fertilization cycles: A prospective clinical study from a tertiary care unit. J Human Repro Scie. 2011;4(1):29.

Cite this article as: Shivtare MV, Lad N, Vishwekar $\mathrm{P}$, Shetty P. Uterine scoring system for reproduction scoring correlation with pregnancy rate in infertility patients undergoing intracytoplasmic sperm injection and embryo transfer. Int J Reprod Contracept Obstet Gynecol 2019;8:2867-70. 